\title{
The Role of Premotor Interneurons in Phase-dependent Modulation of a Cutaneous Reflex during Swimming in Xenopus laevis Embryos
}

\author{
Keith T. Sillar and Alan Roberts \\ Department of Zoology, University of Bristol, Bristol BS8 1UG, England
}

\begin{abstract}
Phase-dependent reflex modulation during fictive "swimming" in Xenopus laevis embryos has been examined with intracellular recordings from rhythmically active spinal neurons. (1) At rest, cutaneous trunk or tail skin stimulation evokes EPSPs in motoneurons and premotor excitatory and inhibitory interneurons of the opposite motor system. During swimming, these EPSPs can only be evoked during the depolarized phase of activity and can then produce extra action potentials that lead to phase-dependent reflexes in ventral roots. On the stimulated side, IPSPs are evoked in rhythmic neurons that can block centrally generated action potentials if the stimulus coincides with the inhibited phase of the swimming cycle. This inhibition suppresses ventral root discharge in a phase-dependent manner. (2) The presence of premotor interneurons in the crossed reflex pathway suggests two parallel routes for cutaneous excitation to reach the motoneurons, one direct and the other indirect through excitatory premotor interneurons. During swimming, the crossed excitation through both routes is gated by the rhythm-generating circuit to allow summation in motoneurons only during the depolarized phase of the swim cycle. (3) Following phasedependent reflexes, the frequency of swimming is raised for several cycles, a phenomenon that requires sensory activation of premotor rhythm-generating interneurons. The results provide evidence on the role of identified premotor spinal interneurons in phase-dependent reflex modulation.
\end{abstract}

The traditional view of the reflex arc as a hard-wired central circuit designed to elicit a stereotyped motor response has changed radically in recent years. Reflexes are often modulated within the CNS so that they suit the behavioral context in which they occur (Duysens, 1977; Forssberg et al., 1977; Grillner et al., 1977; Schomburg and Behrends, 1978; Stehouwer and Farel, 1981; Reichert et al., 1985; Skorupski and Sillar, 1986; Lennard and Hermanson, 1987; Sillar and Roberts, 1988a; Stein and Capaday, 1988; Duysens et al., 1990). Thus, for example, stretch or resistance reflexes that are important in the maintenance of posture but that could impede locomotion if they were

\footnotetext{
Received Apr. 5, 1991; revised Nov. 21, 1991; accepted Nov. 25, 1991.

This work was supported by grants from the MRC. and SERC. K.T.S. is currently a Royal Society University Research Fellow. We thank our colleagues, Drs. S. R. Soffe, W. J. Heitler, T. J. Wiens, and A. J. Simmers, for constructive criticism of earlier drafts of the manuscript and Mrs. L. Teagle for excellent technical assistance.

Correspondence should be addressed to Keith T. Sillar, Gatty Marine Laboratory, Department of Biology and Preclinical Medicine, University of St. Andrews, St. Andrews, Fife KY16 8LB, Scotland.

Copyright (C) 1992 Society for Neuroscience $0270-6474 / 92 / 121647-11 \$ 05.00 / 0$
}

to occur at the wrong moment in the movement cycles are centrally suppressed and may even be reversed in sign in a phasedependent manner (Bässler, 1976; DiCapprio and Clarac, 1981; Skorupski and Sillar, 1986). For monosynaptic reflex arcs, this cycle-by-cycle modulation can occur both presynaptically at the afferent terminals (Baev and Kostyuk, 1982; Sillar and Skorupski, 1986; Dubuc et al., 1988), where GABAAergic inhibition appears to be involved in inhibiting sensory synaptic transmission (Eccles et al., 1963; Davidoff, 1972), and also postsynaptically at the level of the motoneurons themselves (Jordan, 1983).

Most reflex pathways are more complex, for example those involving cutaneous afferent fibers, because one or more sets of interneurons are interposed between the afferents and the motoneurons. Consequently, the number of central sites at which reflex modulation may occur is greater. Four potentially important levels in the nervous system have been recognized (Sillar, 1989) (1) presynaptically at the afferent terminals, (2) postsynaptically at the sensory interneurons that the afferents excite, and postsynaptically at (3) premotor and (4) motoneuronal levels. Evidence on the role of interneurons in central reflex modulation is sparse and has generally been obtained from "simpler" invertebrate systems, in which the interneurons are accessible to intracellular recording and can be identified neuroanatomically and physiolugically (Reichert and Rowell, 1985; Reichert et al., 1985). Comparable information for the vertebrates has been less forthcoming, largely due to the complexity of the central circuitry involved in sensorimotor integration, and the difficulties inherent in recording and characterizing the interneurons. However, there is evidence that $1 \mathrm{a}$ inhibitory interneurons, which are interposed between the motoneurons and inputs from other sources, also receive modulatory input from the spinal locomotor central pattern generator and could therefore gate reflex pathways according to phase (Fcldman and Orlovsky, 1975; Pratt and Jordan, 1987). In addition, the facilitation of afferent excitation to cat motoneurons on the active phase of the locomotor cycle has been interpreted as evidence for the interpolation of "last-order" premotor interneurons in the pathway from cutaneous limb afferents to flexor digitorum longus motoneurons (Schmidt et al., 1988). Functionally, this organization of cutaneous pathways would provide a mechanism for enhancement of inputs to motoneurons from cutaneous sources on particular phases of the movement cycle, but the identity of the interneurons involved has yet to be established.

One simpler vertebrate preparation offering the prospect of direct evidence on the identity and role of interneuronal mechanisms is the swimming system of Xenopus laevis embryos (for review of swimming, see Roberts et al., 1986). The neuroanatomical simplicity of the embryo's spinal cord, containing only 
eight classes of differentiated spinal neurons, has greatly facilitated physiological studies. Recent research has presented evidence on the morphology and role of a number of spinal neuron classes involved in both the generation of the swimming rhythm (Soffe et al., 1984; Dale, 1985; Dale and Roberts, 1985; Soffe, 1987), and the sensory pathways where skin sensory RohonBeard (RB) neurons (RB neurons provide the only known sensory input to the spinal cord; Clarke et al., 1984) directly excite a population of sensory interneurons with crossing axons (Clarke and Roberts, 1984; Sillar and Roberts, 1988a,b; Roberts and Sillar, 1990). These dorsolateral commissural (DLC) interneurons may in turn excite motoneurons on the opposite side (Roberts and Sillar, 1990) to produce a crossed flexion reflex that is usually followed by swimming. We have recently described how phasic inhibition from the central pattern generator for swimming gates the cutaneous excitation of DLC interneurons (i.e., at level 2) so that the crossed reflex excitation that they produce only occurs during the depolarized, post-spike phase of the motoneuron swim cycle (Sillar and Roberts, 1988a; Roberts and Sillar, 1990). Thus, extra motor discharge on the opposite side occurs at the time when it can enhance ongoing contraction to hend the tadpole away from the stimulated side. When extra motor discharge occurs on the opposite side to stimulation, inhibition is seen on the stimulated side that can block motoneuron impulses and avoid conflict between reflex and motor commands. This inhibition suggested that DLCs also excited premotor inhibitory interneurons.

This background information placed us in a position in the present article to examine the gating of motoneuron activity by alternating excitation and inhibition during rhythmic activity in more detail (level 4) and also to investigate the identity and role of premotor spinal interneurons (level 3 ) in cutaneous reflexes during fictive locomotion.

\section{Materials and Methods}

All experiments were performed on Xenopus laevis embryos at developmental stage 37/38 (Nieuwkoop and Faber, 1956) at room temperature. Methods have been described in detail elsewhere (Sillar and Roberts, 1988a,b; Roberts and Sillar, 1990), but briefly, embryos were removed from their egg membranes, immobilized in 70-100 $\mu \mathrm{M}$ D-tubocurarine chloride (Sigma) and secured on their sides to the Sylgard surface of a Perspex platform located within a $2 \mathrm{ml}$ preparation bath. The platform could be rotated about its long axis to facilitate dissection and recording. Preparations were continuously superfused with frog Ringer's containing tubocurarine of the following ionic composition (in $\mathrm{mm}$ ): $\mathrm{NaCl}, 120 ; \mathrm{KCl}, 2.5 ; \mathrm{CaCl}, 5 ; \mathrm{NaHCO}_{3}, 15$ (pH 7.6). The Ringer's was continuously bubbled with $95 \% \mathrm{O}_{2} / 5 \% \mathrm{CO}_{2}$.

The trunk skin overlying the myotomal muscles on the left side was peeled back from the level of the otic capsule to the level of the anus and removed with the aid of fine etched tungsten needles. Extracellular recordings of motoneuron activity were made with glass suction electrodes placed over ventral roots in the intermyotome clefts (cf. Kahn and Roberts, 1982). The myotomes overlying the rostral spinal cord were also removed from the otic capsules to the mid-trunk region, to enable intracellular recording from the exposed spinal cord. Neurons were impaled with glass microelectrodes either using capacity overcompensation or with the aid of a piezoelectric jolting device (Weevers, 1972). The following criteria were adopted for "acceptable" penetrations: neurons had stable resting potentials of $<-60 \mathrm{mV}$; they displayed clear synaptic drive during swimming, with phasic on-cycle EPSPs that could trigger impulses and mid-cycle IPSPs; they fired impulses of 30$60 \mathrm{mV}$ during swimming and following depolarizing current injection. Microelectrodes were filled with either $3 \mathrm{~m}$ potassium acetate $(\mathrm{pH} 7.4$; DC resistances, $150-350 \mathrm{M} \Omega$ ) or with a $5 \%$ solution of Lucifer yellow (Stewart, 1978) in $3 \mathrm{M} \mathrm{LiCl}$ (10 preparations). Lucifer electrodes had DC resistances of $>1000 \mathrm{M} \Omega$ before being beveled to around $400 \mathrm{M} \Omega$. Neurons were filled with Lucifer yellow iontophoretically using $0.5 \mathrm{sec}$, $0.1 \mathrm{nA}$ pulses every second for up to $10 \mathrm{~min}$. Spinal cords containing
Lucifer-filled neurons were fixed in $4 \%$ formalin in phosphate buffer, processed conventionally through an alcohol series (Langs), cleared in methyl benzoate, and viewed as whole-mounts under an Olympus epifluorescence microscope.

Rohon-Beard (RB) sensory neurons (Hughes, 1957) provide the only known innervation of the trunk skin at this stage in development (Roberts and Hayes, 1976; Clarke et al., 1984). For this reason, the peripheral neurites of RB neurons could be stimulated specifically using a glass suction electrode ( $50 \mu \mathrm{m}$ tip opening) placed on the left and/or right tail skin about the level of the anus (Clarke et al., 1984). Current pulses ( 0.5 or $1 \mathrm{msec}$ duration) were applied to the opposite side to evoke excitatory postsynaptic potentials (EPSPs) in ventrally positioned neurons, presumed to be motoncurons, and in rhythmic interneurons. Stimuli applied to the same side evoked IPSPs in rhythmic neurons. To examine the modulation of these potentials during rhythmic motor output, stimuli at an intensity sufficient to evoke relatively constantamplitude PSPs were applied repetitively at 1 or $2 \mathrm{~Hz}$ and episodes of rhythmic fictive swimming activity were then evoked usually through the pineal sensory pathway by dimming the illumination (Roberts, 1978) or by a higher-intensity stimulus applied elsewhere on the skin. Conventional recording and amplification techniques were used throughout. Signals were stored on a Racal tape recorder, and permanent records were made using a Gould digital oscilloscope coupled to an X-Y plotter. The results presented here are selected from 37 intracellular recordings of rhythmically active interneurons located in the dorsal half of the cord and from six ventral neurons, presumed to be motoneurons in $43 \mathrm{em}$ bryos. Five of the dorsal rhythmic neurons, from a total of 10 preparations, were successfully filled with Lucifer yellow.

\section{Results}

Phase-dependent modulation of contralateral excitation and ipsilateral inhibition during swimming: distributed effects onto motoneurons

We have shown previously that the excitation of intracellularly recorded ventral neurons following stimulation of the skin on the opposite side is modulated during swimming activity in a phase-dependent fashion (Sillar and Roberts, 1988a). The excitatory pathway from the opposite side will lead to a reflex action potential in ventral neurons if the stimulus is delivered at phase values in the swimming cycle when the neurons are already depolarized (the active phase). Stimuli falling in the opposite half of the rhythm, when neurons receive mid-cycle inhibition, evoke no detectable responses in the motoneurons. The IPSPs evoked by stimulation on the same side as the ventral neurons were also able to block the centrally driven action potentials if they were evoked at the appropriate phase in the rhythm, when the neurons were already inhibited during swimming.

It is not possible to establish motoneuron identity by antidromic ventral root stimulation, but we assumed that the majority of ventral neurons would have been motoneurons since anatomical studies have shown that the ventral cord is predominantly occupied by motoneuron somata (Roberts and Clarke, 1982). To establish conclusively that the reflex modulation of unidentified neurons reflected the phase-dependent modulation of motoneurons, we have examined the modulation of yentral root impulses when the skin is stimulated at different phases of the swimming cycle. The results from six experiments have confirmed that ventral rout (motoneuron) activity is phasically modulated during swimming activity (Figs. 1, 2; see also Figs. $4,7,10$ ). Stimuli to the opposite side that coincide with the depolarized phase of activity reliably evoked reflex ventral root discharge on 24 out of 27 occasions (e.g., Fig. 1A). Similarly, the inhibition of motoneurons following skin stimulation on the same side that prevents intracellularly recorded action potentials during swimming in a phase-dependent way was correlated with a blockade of ventral root activity on seven out of nine occasions 
A

opposite side
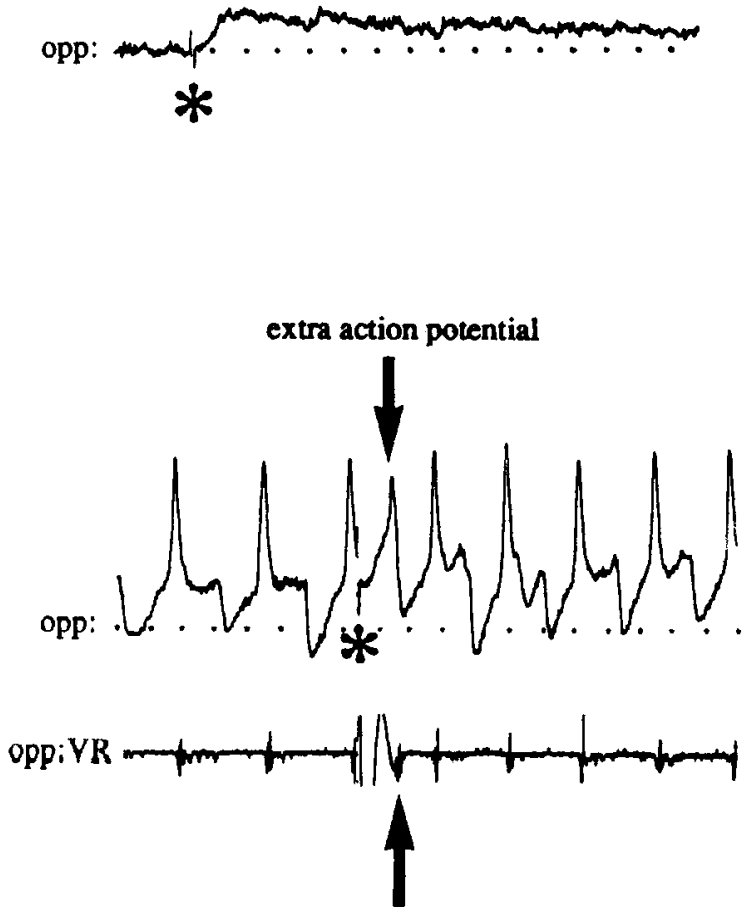

B

stimulated side

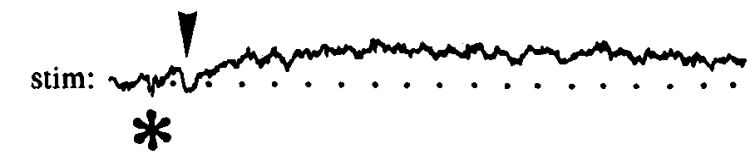

$20 \mathrm{mV}$

$100 \mathrm{msec}$

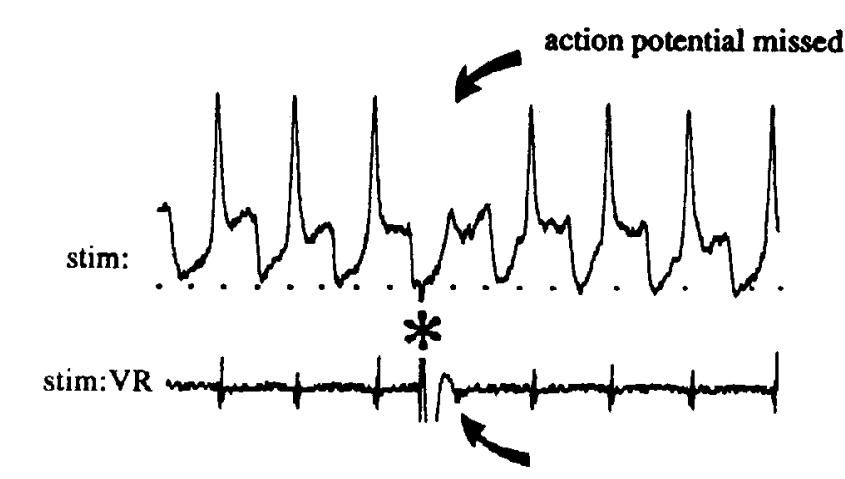

Figure 1. Phase-dependent modulation of afferent input to motoneurons. $A$, Skin stimulus $\left(^{*}\right)$ evokes EPSP in opposite ventral rhythmic neuron (opp:). Same stimulus strength applied during swimming evokes extra action potential in neuron and reflex ventral root (opp:VR) discharge (at lower arrow) if stimulus occurs during depolarized phase. B. Stimulus evokes short-latency IPSP in neuron on stimulated side (stim:; at arrowhead). Stimulus during swimming suppresses centrally generated excitation and action potential is missed (stim:, upper arrow). Ventral root discharge is also suppressed (stim:VR; lower arrow indicates expected timing of ventral root spike based on cycle preceding stimulus). Dotted line in this and subsequent figures represents resting potential prior to onset of swimming.

(Fig. 1B). Fourteen stimuli at inappropriate phases of the swimming cycle had no effect either on intracellular activity or on the ventral root. This approach has also allowed us to assess whether reflex modulation is a local or a distributed phenomenon, since the extracellular ventral root recordings were always a significant distance away from the intracellularly recorded rhythmic ventral neurons. The ventral rhythmic neuron shown in Figure 2 was penetrated at the level of the 4th postotic cleft while the extracellular ventral root recording was made at the 8 th clcft. In the example shown in Figure 2, simultaneous recordings were made from a ventral rhythmic neuron at the 4th postotic cleft and from ventral roots at the 8 th and $14 \mathrm{th}$. Stimulation on the same side led to an IPSP that suppressed the centrally driven action potential at all three levels in the cord. These data suggest that the phase-dependent modulation of motoneuron activity is distributed along the length of the spinal cord.

\section{Longer-term effects of reflex activation during swimming imply activation of premotor interneurons}

Stimulation of the skin at phases in the swimming cycle when phase-dependent reflexes lead to extra or missed action potentials in motoneurons also results in marked changes in the frequency and timing of the swimming rhythm. An example, recorded from a rhythmic ventral neuron, is shown in Figure 3. In response to a skin stimulus on the opposite side at rest a subthreshold EPSP was recorded (Fig. $3 \mathrm{Ai}$ ). During fictive swimming activity, the same intensity of stimulation evoked an extra action potential within the same cycle, so long as the stimulus occurred during the depolarized, post-spike phase (Fig. 3Aii). However, three other notable effects can be observed. First, the frequency of the swimming rhythm increases for several subsequent cycles (Fig. 3B, upper graph). In a sample of 46 responses in 13 neurons occurring in the appropriate phase following contralateral stimulation, frequency increased obviously in $89 \%$ of occasions. Sccond, the increase in frequency, which lasts for about $500 \mathrm{msec}$ or approximately the duration of the underlying EPSP, is accompanied by a rise in the level of tonic depolarization upon which the motoneuron activity rests (Fig. $3 B$, lower graph; measured from resting potential before onset of swimming to depolarized level just prior to onset of midcycle IPSP; $85 \%$ of 46 contralateral stimuli). The amount of change in tonic depolarization was variable and in some cases was minimal, despite a clear increase in frequency (e.g., Fig. $4 B$ ). Third, the rhythm is often reset to the timing of the reflexively driven action potential (Figs. $3 \mathrm{Aii}, 4 ; 85 \%$ of 46 contralateral stimuli) and the first rhythmic IPSP to follow the stimulus is phase-delayed when compared to its expected timing based on the prestimulus rhythm, showing that the rhythm on the opposite side is also reset.

Similar results were obtained in response to ipsilateral stimuli. In the ventral neuron recorded in Figure 5, stimulation on the same side evoked an IPSP (Fig. 5A,C). When an appropriately 


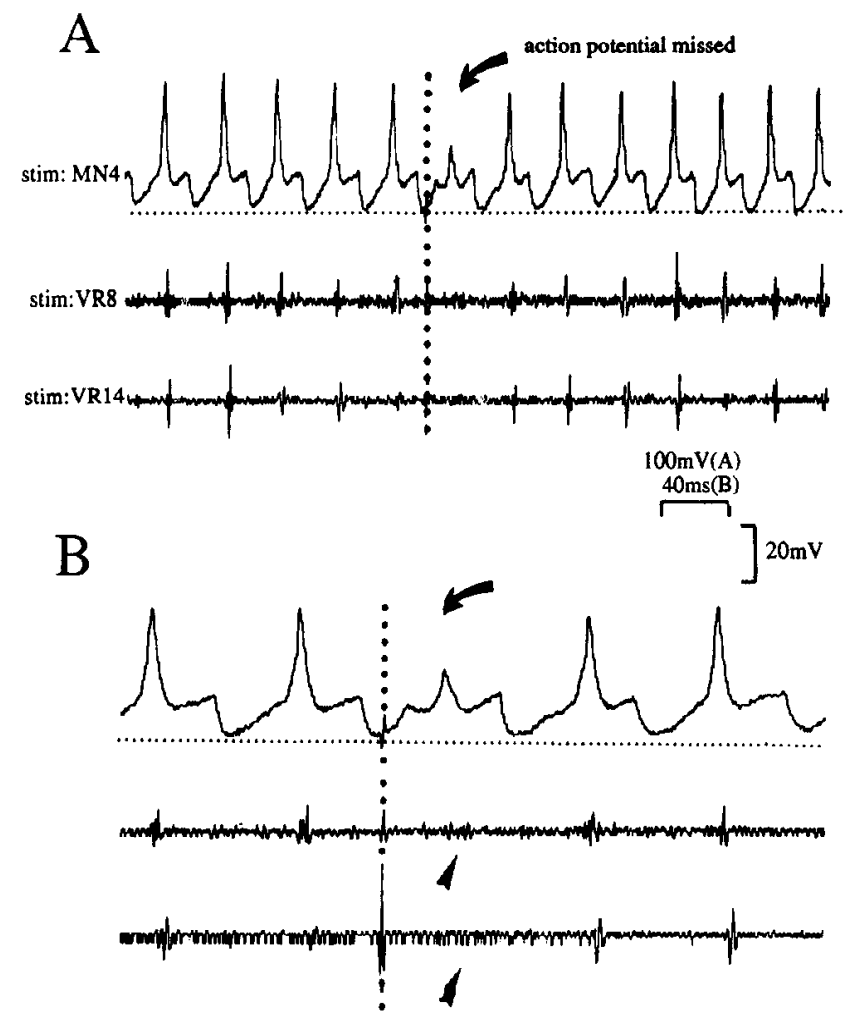

Figure 2. Phase-dependent reflex modulation is distributed longitudinally throughout motor system. $A$, Excerpt of fictive swimming with intracellular recording from ventral neuron, presumably a motoneuron, on stimulated side level of 4th postotic cleft (stim:MN4) and ventral root recordings on stimulated side at 8th (stim:VR8) and 14th (stim: $V R 14$ ) postotic clefts. Note brief rostrocaudal delay between recording sites. Skin stimulus on same side during inhibited phase (at vertical dotted line) evoked IPSP at arrow to suppress intracellular action potential and ventral root discharge. $B$, Expansion of $A$ to show suppression of activity at three recording sites. Arrowheads indicate expected timing of ventral root activity based on cycle preceding stimulus (at vertical dotted line). Note increased frequency of poststimulus rhythm.

timed stimulus of the same intensity was delivered during swimming, it suppressed the centrally driven action potential but then led to an increase in tonic depolarization and rhythm frequency (Fig. 5B) (ca. 15\%) and an increase in the amplitude of the mid-cycle IPSPs (in this case, the rhythm was not obviously reset; in a sample of 20 responses in eight neurons to stimuli in the appropriate phase, resetting occurred on $90 \%$, increased rhythm frequency on $80 \%$, and an increase in tonic depolarization on $90 \%$ of responses).

In Xenopus, there is no evidence that spinal motoneurons have synaptic outputs in the cord, and as in the lamprey (Wallén and Lansner, 1984), they appear to be followers of premotor intermeluronal drive and are not part of the rhythm-generating network. The effects on the timing and frequency of the rhythm generator for swimming that follow phase-dependent reflexes (cf. Figs. 3-6) therefore imply phase-dependent activation of premotor excitatory and inhibitory rhythm generating interneurons.

\section{Cutaneous input to dorsal rhythmic interneurons and its phase modulation}

To obtain evidence that the crossed cutaneous pathway from RB cells excites premotor interneurons, we recorded intracel-
A

stimulus on opposite side
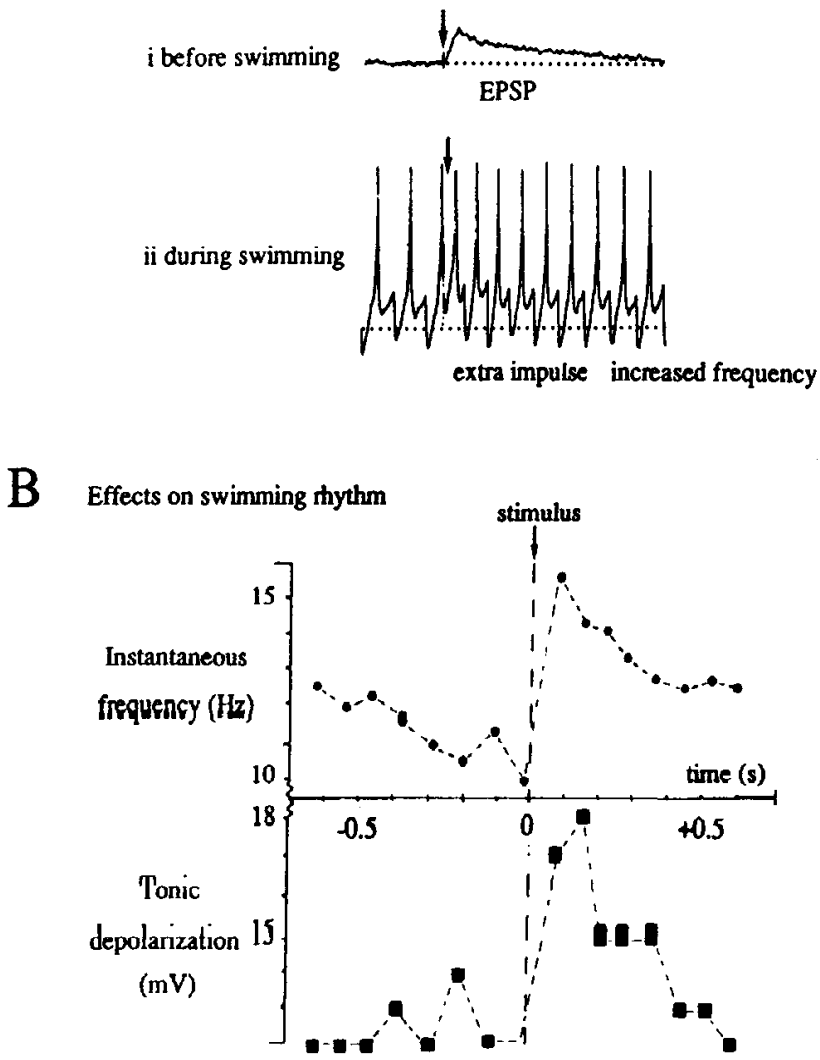

Figure 3. Phase-dependent reflexes modulate the output of the central rhythm generator for swimming. $A i$, Stimulus on opposite side at rest evokes excitation in a ventral neuron. Aii, During swimming, stimulus at the same intensity evokes an extra action potential, if it occurs during the post-spike, depolarized phase of the swimming cycle. Following the extra action potential, the swimming rhythm is reset to the timing of response (see also Fig. 4), lhe frecuency of the swimming rhythm increases, and the tonic depolarization is raised for several subsequent cycles. $B$, Graphic representation of the effects of one such stimulus (arrowed, time zero) on instantaneous swimming frequency (upper graph) and tonic depolarization (lower graph). Note that both parameters broadly parallel the time course of the underlying $\operatorname{EPSP}(A i)$.

lularly from 37 neurons in the dorsal half of the spinal cord that were rhythmically active during fictive swimming. In this location, motoneurons are sparse (Roberts and Clarke, 1982), so the recorded neurons were likely to be premotor interneurons. Dorsal rhythmic neurons were excited at short latency by skin stimulation on the opposite side (Fig. 6). The EPSPs could normally be graded in amplitude with stimulus intensity, though only rarely was the excitation large enough to evoke an action potential in the neuron. More usually, swimming activity was evoked at stimulus intensities that resulted in only subthreshold short-latency excitation. The EPSPs occurred at latendies that ranged from 8 to $18 \mathrm{msec}$ [mean, $12.31 \pm 2.19 \mathrm{msec}( \pm \mathrm{SD}) ; n$ $=71$ from 7 neurons]. The range and mean latency were not significantly different from EPSPs evoked in a similar way in ventral neurons, presumably motoneurons [Fig. $6 B, 12.29 \pm$ $1.84 \mathrm{msec}( \pm \mathrm{SD}) ; n=77$ from 10 neurons; Roberts and Sillar, 1990], suggesting that the crossed excitatory pathway makes parallel connections onto both motoneurons and premotor interneurons.

Like the synaptic excitation of ventral neurons, the EPSPs 


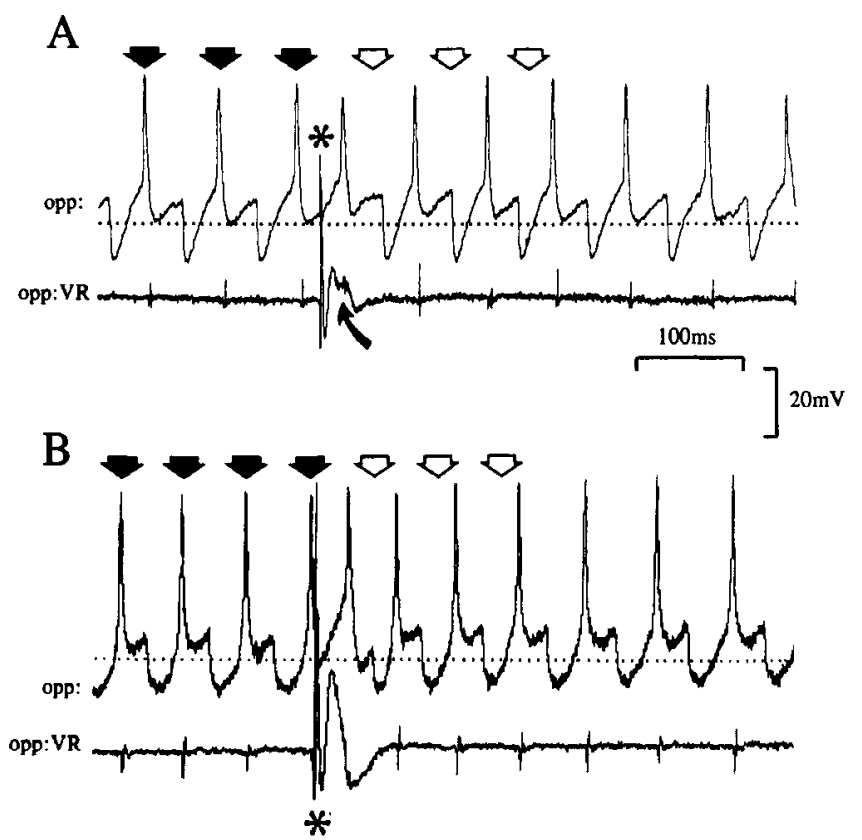

Figure 4, Phase-dependent reflexes reset timing of central rhythm generator for swimming. $A$ and $B$. Two examples from same cxperiment: intracellular recording from ventral rhythmıc neuron ( $O p p^{\circ}$ ) and ventral root recording (opp:VR). Stimuli on the opposite side $\left({ }^{*}\right)$ evoke extra action potential and ventral root discharge (curved arrow) if they occur during the depolarized phase of the cycle. Poststimulus rhythm is reset to timing of reflex action potential. Solid arrows show timing of prestimulus rhythm. Open arrows represent expected timing of action potential based on cycle before stimulus. See also Figure 3 Aii.

evoked in rhythmic dorsal neurons by skin stimulation on the opposite side were modulated in a phase-dependent way during swimming (Fig. 7). Stimuli that evoked an EPSP in the quiescent embryo (Fig. 7A) evoked no response if the stimulus occurred during the hyperpolarized phase of the swimming cycle (Fig. $7 B$ ) but usually evoked an extra action potential if they coincided with the depolarized plateau of activity (Fig. $7 C, D$ ). These data suggest that premotor interneurons, those that are responsible for the generation of the swimming rhythm, can be recruited by the sensory pathway to increase their firing at certain phases of the swimming rhythm.

The majority of dorsal rhythmic neurons ( 8 of 10 tested) also received short-latency inhibitory synaptic potentials in response to skin stimulation on the same side (Fig. 8A,C). As for motoneurons, the IPSPs were able to suppress centrally driven action potentials if they occurred in the hyperpolarized phase of the rhythm (Fig. 8B).

The ability of sensory stimuli to induce phase-dependent modulation of impulse activity was robust. In 10 of the interneurons examined in detail, $83 \%$ ( 90 of 109) of stimuli occurring in the appropriate phase (depolarized phase for contralateral stimuli, inhibited phase for ipsilateral stimuli) enhanced or supressed spiking, respectively. In 15 of these examples, contralateral stimulation in the depolarized phase failed to induce an extra action potential but did evoke a phase-dependent EPSP and a subsequent increase in the frequency of the swimming rhythm. In contrast, stimuli falling at inappropriate phases failed to induce any synaptic response or change in swimming frequency in $94 \%$ (67 of 71 ) of cases.
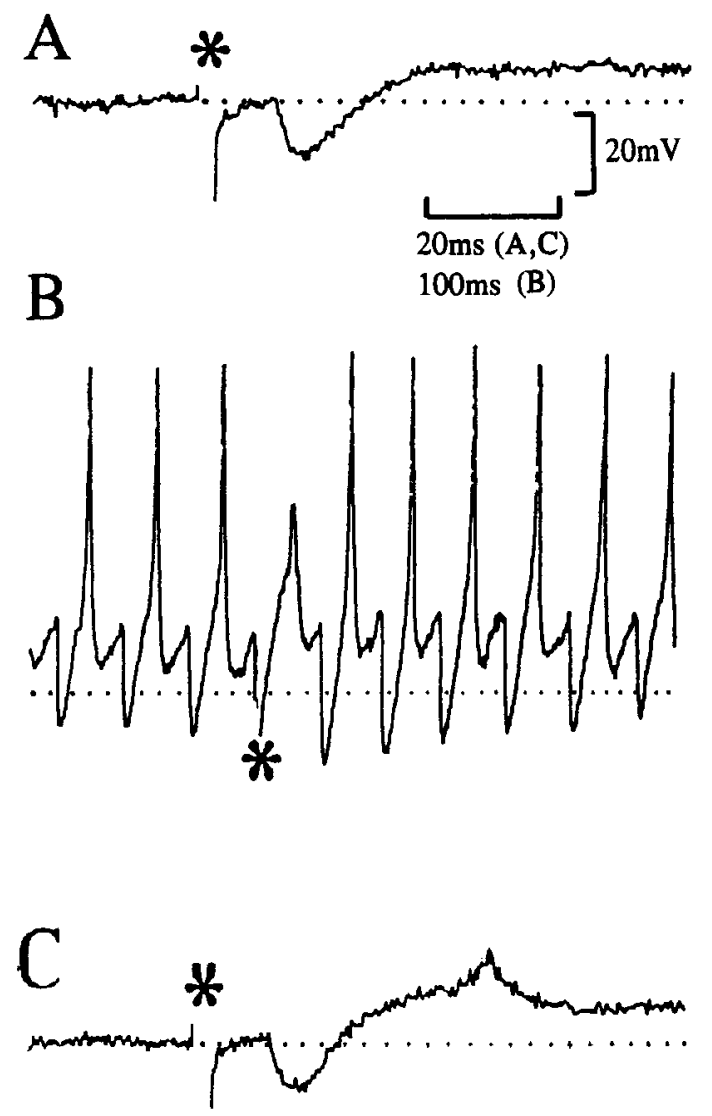

Figure 5. Phase-dependent effects on rhythm generation in a rhythmic ventral neuron following skin stimulation on the same side. $A$, Stimulus $\left.{ }^{*}\right)$ evokes short-latency IPSP followed by excitation. Dots are resting potential before stimulus. $B$, During swimming the same stimulus strength during the inhibited phase suppresses centrally driven action potential. In poststimulus rhythm note slight increase in frequency, mid-cycle IPSP amplitude, and tonic depolarization. $C$, Stimulus after swimming episode still evokes IPSP.

\section{Modulation of synaptic input to identified premotor interneurons}

Two classes of spinal interneurons appear to be directly involved in rhythm generation in the embryo: excitatory interneurons that we presume to be descending interneurons (Dale and Roberts, 1985; Roberts and Alford, 1986) and inhibitory commissural interneurons (Dale, 1985). Both types are rhythmically active during swimming, displaying the same form of activity seen in motoneurons. We have sought direct evidence, using dye-filled microelectrodes, on the identity of the dorsally positioned rhythmically active interneurons that are excited by stimulation on the opposite side. Three descending interneurons and two commissural interneurons were successfully recorded and stained intracellularly with Lucifer yellow.

An example of a commissural interneuron is shown in Figure 9. The interneuron had anatomical features characteristic of the commissural interneurons that have previously been shown to mediate reciprocal inhibition of contralateral neurons (Dale, 1985 ) and by immunocytochemical techniques to show glycinelike immunoreactivity (Dalc ct al., 1986; Roberts et al., 1988). The soma was located in the dorsal half of the rostral spinal cord, and the ventrally projecting neurite sprouted numerous dendrites in the spinal marginal zone before crossing in the ventral commissure and bifurcating into short rostral and caudal 

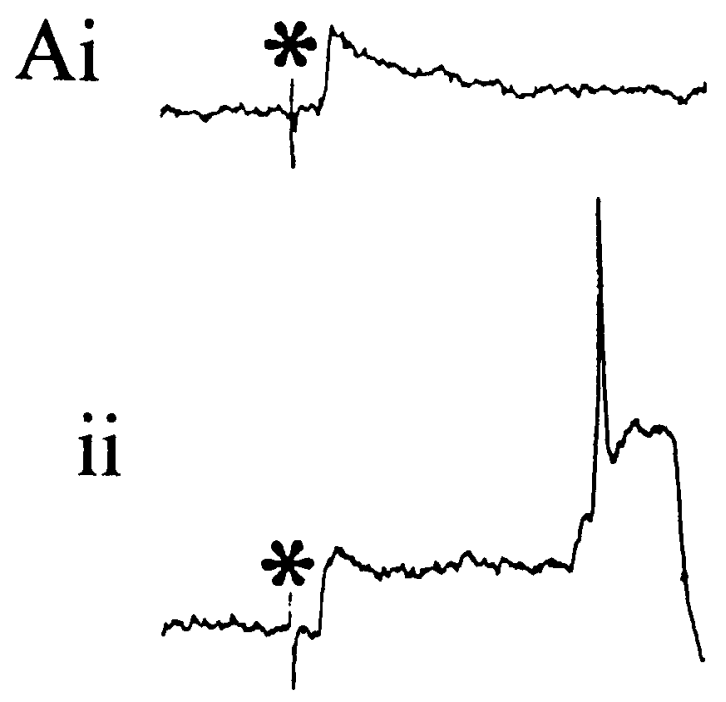
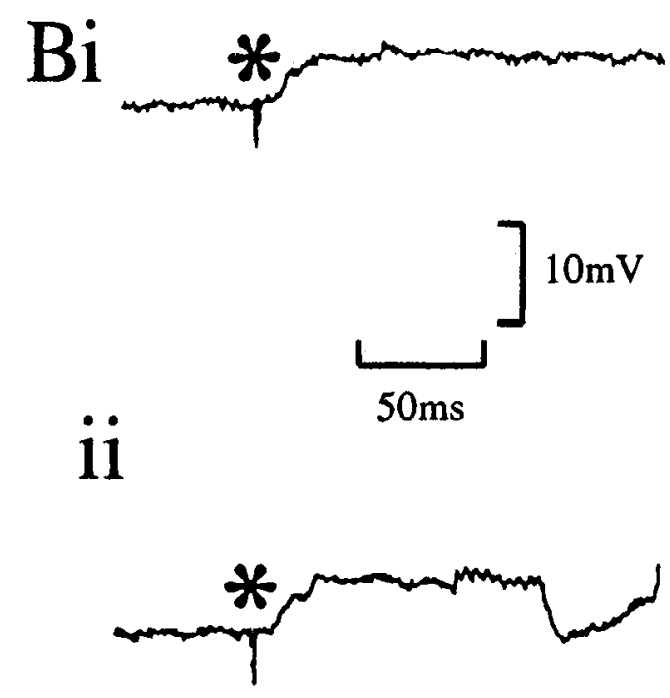

iii

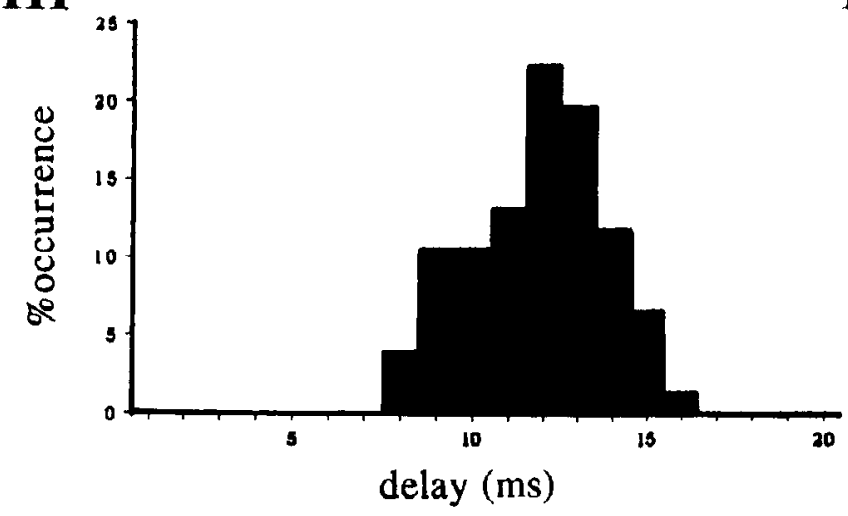

iii

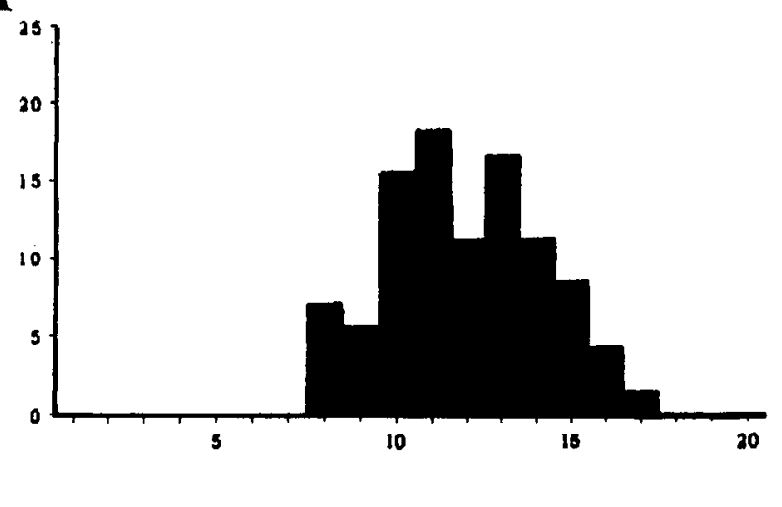

Figure 6. Evidence for parallel crossed activation of dorsal and ventral rhythmic neurons. The traces show EPSPs in response to skin stimulation on opposite side just below (i) and just above (ii) swimming threshold recorded in a dorsally positioned neuron (Ai and $A i i)$ and a ventrally positioned neuron $(B i$ and $B i i)$. The two neurons were recorded in the same preparation. Aiii and Biii, Histograms of latencies to EPSPs evoked by stimuli on the opposite side in dorsal (Aiii) and ventral (Biii) neurons. Aiii. Delays to onset of 71 contralateral EPSPs recorded in seven dorsal rhythmic neurons. Mean delay, $12.31 \pm 2.19 \mathrm{msec}( \pm$ SD). Biii, Delays to onset of 77 contralateral EPSPs recorded in 10 ventral rhythmic neurons. Mean, $12.27 \pm 1.84 \mathrm{msec}( \pm \mathrm{SD})$. Note similar range and mean latencies.

axonal projections on the opposite side of the cord. The interneuron was rhythmically active (Fig. $9 B, D$ ), discharging impulses in time with ipsilateral ventral root spikes during swimming, although when swimming frequency declined toward the end of an episode it frequently did not spike on each cycle of activity. Siimulation of the skin on the opposite side relizbly evoked subthreshold excitation (Fig. 9C) with a time course similar to that recorded in unidentified dorsal rhythmic neurons, and if the neuron was depolarized by intracellular current injection, the EPSP could evoke an action potential. During swimming activity, the excitation was modulated in a phase-dependent way, similar to the modulation of sensory excitation in other rhythmic neurons: if the stimulus coincided with the inhibitory phase of the swimming rhythm, no response could be detected and the rhythm continued unaltered (Fig. 9Dii). However, when the stimulus coincided with the excitatory phase of the activity, excitation appeared and could trigger an extra ac- tion potential in the commissural neuron (Fig. 9Di). Thus, the crossed excitation of the interneuron was rhythmically modulated during swimming activity in the same way as ventrally positioned motoneurons.

Essentially the same results were obtained for descending interneurons. In the example shown in Figure 10, an interneuron was recorded at the 4 th postotic myotome that had anatomical features characteristic of the descending interneurons described on the basis of HRP fills (Roberts and Clarke, 1982; Roberts and Alford, 1986) and of an excitatory interneuron described by Dale and Roberts (1985) based on paired recordings from an interneuron and motoneuron on the same side of the cord. The interneuron (Fig. 10A) was rhythmically active during swimming (Fig. 10B,D) and was excited following skin stimulation on the opposite side (Fig. 10C). The excitation was modulated at different phases of the rhythm again so that the stimulus evoked no response if it coincided with the inhibitory phase 

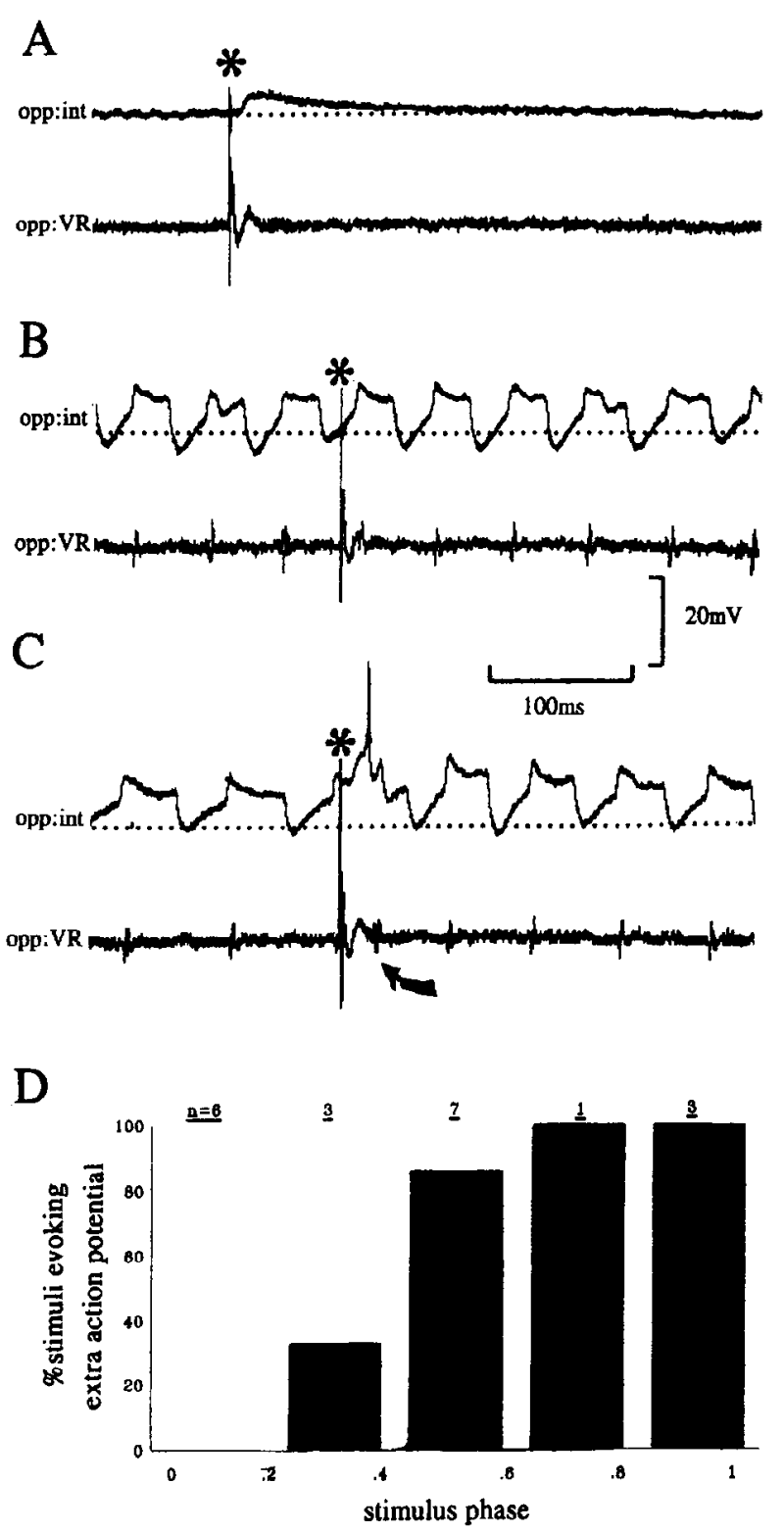

Figure 7. Phase-dependent modulation of crossed excitation to an unidentified dorsal interneuron (opp:int) following stimulus to the opposite side (*). $A$, At rest a subthreshold stimulus evokes EPSP. $B$ and $C$, Same stimulus during swimming evokes no response if delivered during the inhibited phase of cycle $(B)$ but evokes extra action potential in interneuron and ventral root (opp:VR) (at arrow) if delivered during depolarized phase $(C)$. Note poststimulus rhythm reset with increased frequency, tonic depolarization, and mid-cycle IPSP amplitude. $D$, Histogram of percentage stimuli on the opposite side evoking extra action in relation to phase in swim cycle on the stimulated side. Stimuli occurring late in cycle when opposite neurons are depolarized evoke extra action potentials. Twenty data points were taken from seven experiments.

(Fig. 10Dii), but could evoke an extra action potential if it coincided with the depolarized plateau of activity (Fig. 10Di).

\section{Discussion}

\section{Reflex modulation and premotor interneurons}

Our results show that the DLC sensory interneurons make parallel direct connections onto motoneurons, and premotor commissural and descending interneurons on the opposite side of
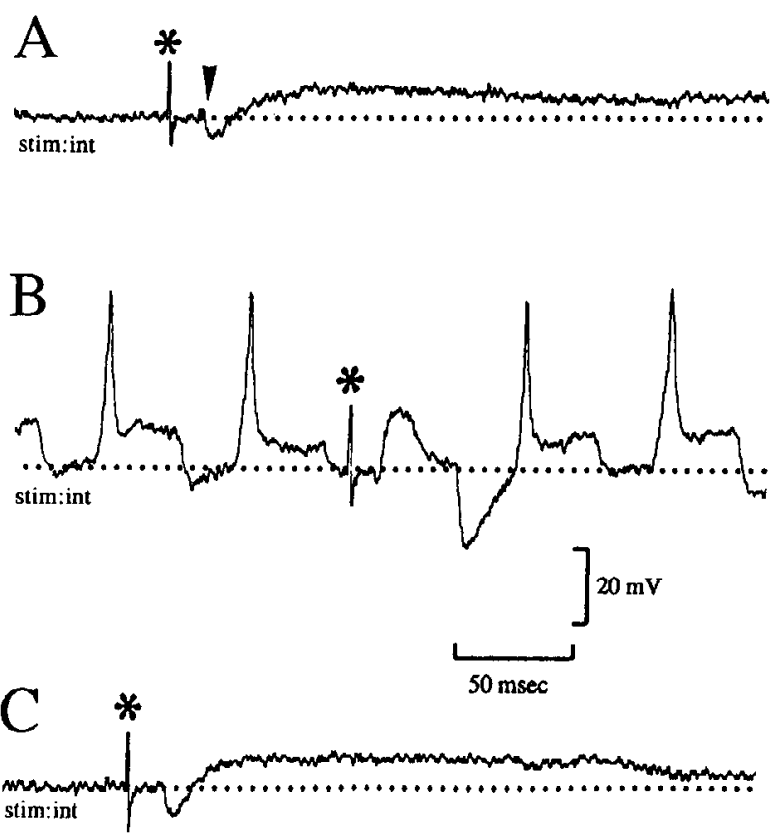

Figure 8, Dorşal rhythmic interneurons are inhibited at short latency by skin stimulation on same side $\left({ }^{*}\right) . A$, Subthreshold stimulation evokes short-latency IPSP (at arrowhead) followed by excitation (cf. Fig. $1 B$ for ventral rhythmic neuron). $B$, During swimming activity, same stimulus suppresses centrally driven action polential if delivered in hyperpolarized phase. In opposite phase, stimulus evoked no response (not shown). $C$, Stimulus shortly after end of episode still evokes subthreshold response.

the cord (Fig. 11A). These interneurons are rhythmically active and receive alternating excitation and inhibition during swimming. The crossed excitation seen in these rhythmic premotor interneurons (level 3 ) was phasically modulated during swimming activity in the same manner as that described earlier for presumed motoneurons (Sillar and Roberts, 1988a). The excitation is powerfully suppressed during the inhibited phase of the rhythmic synaptic input when DLC interneurons are also inhibited. This result is consistent with the DLC interneurons being the source of the underlying excitation and supports our conclusion that the DLC interneurons make parallel output connections onto all rhythmically active contralateral neurons.

We can now ask what role the premotor interneurons could play in modulating cutaneous reflexes during swimming. The experimental protocol that we adopted was to stimulate the skin at an intensity that evoked only subthreshold excitation in rhythmic neurons on the opposite side (Fig. 11A). The inhibited phase of these rhythmic neurons' activity coincides with the inhibited phase in the DLC interneurons so skin stimulation at this time produces no response in the interneuron (Fig. 11B). However, on the depolarized post-spike phase of activity in motoneurons (level 4) and rhythmically active premotor interneurons (level 3 ), the previously subthreshold crossed excitation is able to evoke extra action potentials (Fig. 11C). The gain of the reflex must therefore be enhanced. This suggests that the rhythm generator for swimming plays an important role in modulating the crossed reflex pathway. Inputs that coincide with the depolarized plateau of activity will be more likely to trigger action potentials because for this phase in the rhythm the motoneurons will be closer to spike threshold. In this way, the central rhythm generator for swimming might "gate in" a reflex 

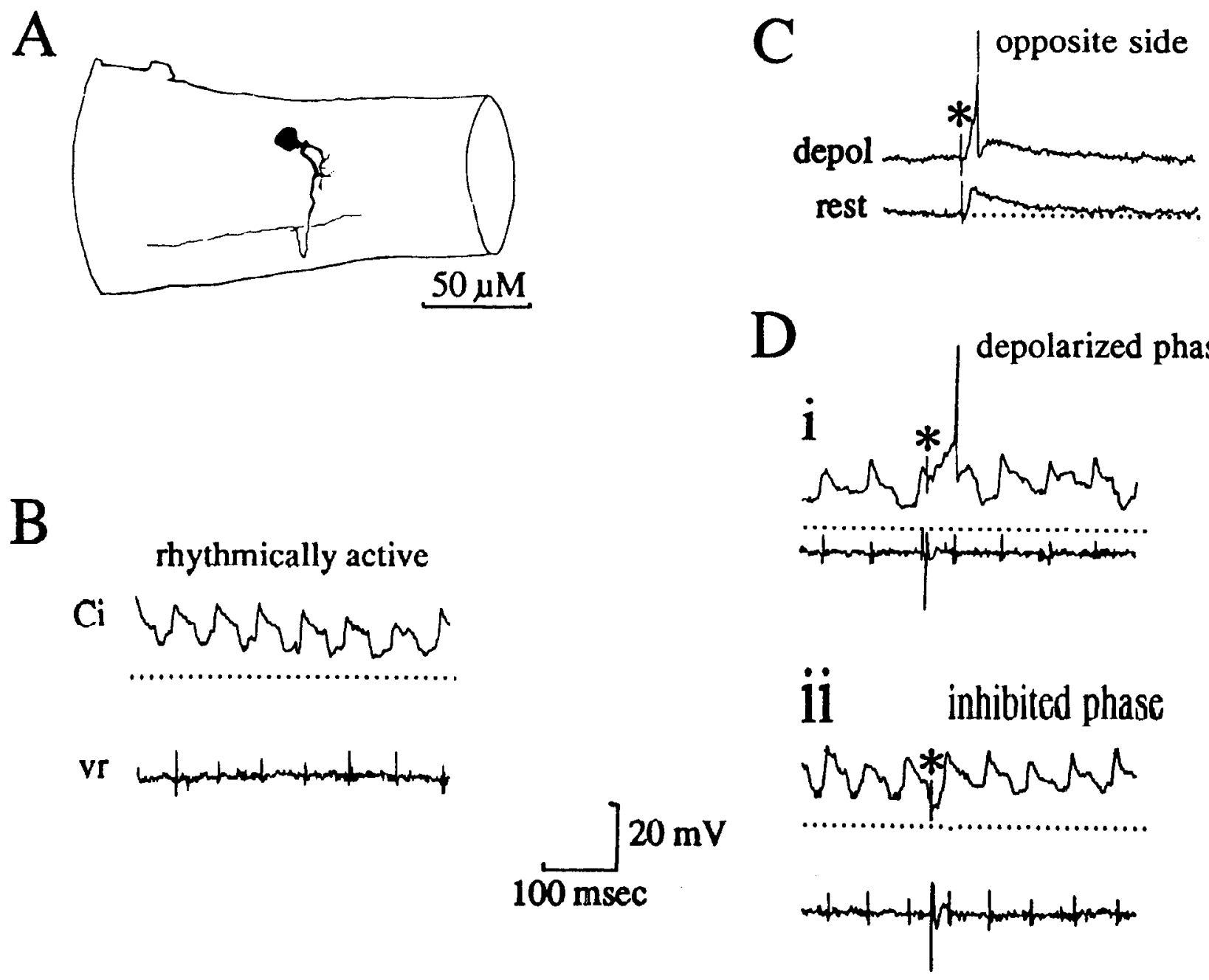

Figure 9. Phase-dependent modulation of crossed excitation in a commissural interneuron $(C i)$. $A$, Camera lucida drawing of interneuron filled with Lucifer yellow (see Materials and Methods). The interneuron was recorded on the left side at approximately the level of the 3rd postotic myotome (rostral, left; dorsal, top). Note dorsal position of soma, ipsilateral processes, and contralateral projection, $\mathrm{T}$ branching to give short axons running rostrally and caudally in contralateral cord. These features are characteristic of inhibitory commissural interncurons (cf. Dalc, 1985; Dale et al., 1987). $B$. The interneuron was rhythmically active during swimming in phase with ventral root (vr) activity on same side as soma, but did not always discharge action potentials. $C$, Skin stimulation on opposite side evokes excitation. Following depolarizing current injection (depol), the EPSP could trigger an action potential. Di and Dii, Same stimulus on opposite side during swimming evoked extra action potential if given during the depolarized phase of the cycle $(i)$, but evoked no response if given during the inhibited phase (ii).

that would not normally have occurred in the stationary or quiescent animal.

The inclusion of the rhythm-generating circuitry in the crossed excitatory pathway may suggest a second means by which the gain of the reflex can be enhanced during swimming. In particular, the crossed excitation of descending excitatory interneurons implies two independent pathways for sensory excitation to reach the motor system (Fig. 11). The first is disynaptic, from $R B$ neurons via DLC interneurons to the motoneurons. The second is more complex with premotor interneurons interposed between DLC interneurons and motoneurons. Of these premotor elements, one set, the descending interneurons, can themselves excite the motoneurons (Dale and Roberts, 1985). In the quiescent embryo, the gain of the second pathway is lower because the descending interneurons sit at their resting potential (Fig. 11 A). However, during the depolarized phase of swimming activity the gain of the pathway is increased and the indirect pathway is now brought into play and can sum with the disynaptic pathway to facilitate the cutaneous excitation of motoneu- rons (Fig. 1 1C). Strong circumstantial evidence suggests that the descending interneurons also excite themselves in a positive feedback loop that may be essential for sustaining the swimming rhythm (Roberts et al., 1986). Hence, phase-dependent activation of the descending interneurons will be likely to lead to excitatory effects on the swimming rhythm itself. On the inhibitory phase of swimming, both pathways are suppressed because spiking in DLC interneurons, the source of the crossed sensory excitation, is blocked by the rhythmic inhibition they receive during rhythm generation (Fig. 11B).

\section{Other animals}

Parallels are emerging with the organization of cutaneous pathways in the spinal cords of Xenopus embryos and adult vertebrates, where there are many examples of phase-dependent modulation of cutaneous reflexes during locomotion (Forssberg et al., 1975, 1977; Duysens, 1977; Grillner et al., 1977; Duysens and Stein, 1978; Schomburg and Behrends, 1978; Forssberg, 1979; Schomburg and Steffens, 1988; Currie and Stein, 1989). 

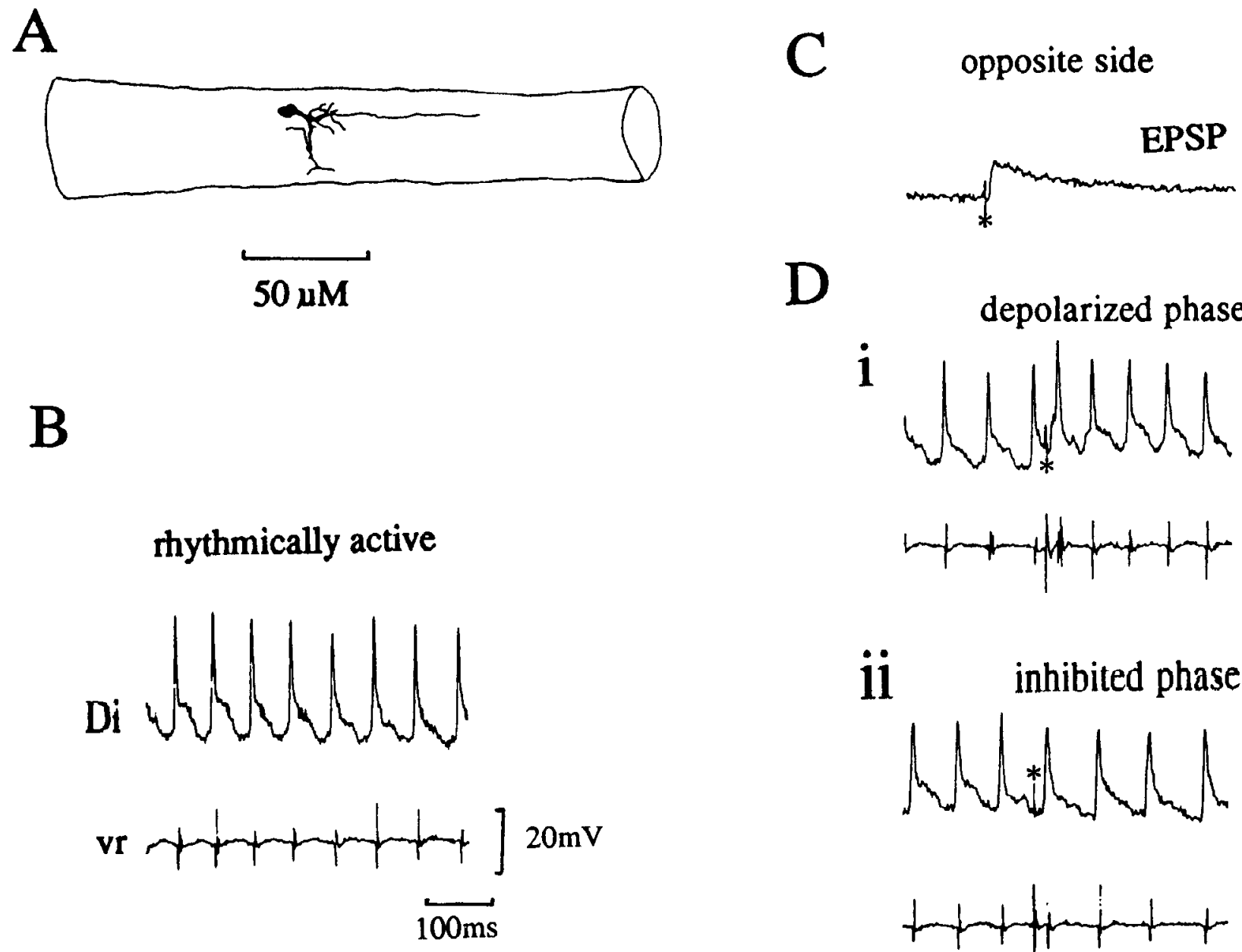

$\mathrm{D}$
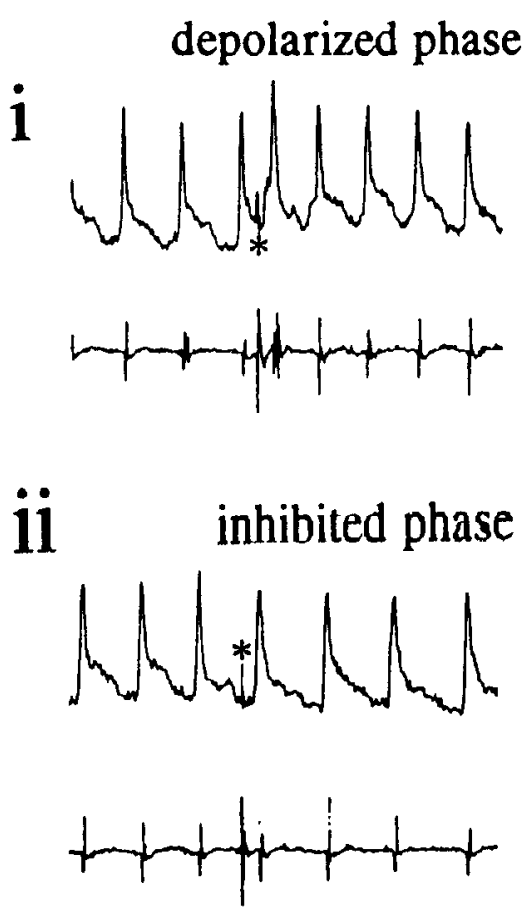

Figure 10. Phase-dependent modulation of crossed excitation to a descending interneuron. The interneuron (Di), which was rhythmically active during swimming in time with ventral root $(v r)$ activity in same side $(A)$ had a dorsally located soma, on left side at approximately the 5 th postotic myotome, ipsilateral dendrites in marginal zone and a descending ipsilateral axon that could be traced for approximately $70 \mu \mathrm{m}$. $C$, Skin stimulation on the opposite side evoked excitation in the interneuron $(E P S P)$. Di and Dii. Stimulation at same intensity as $C$ evoked no response if it occurred in the inhibited phase (ii) but led to an extra action potential if it occurred during the depolarized phase (i). Note reflex on VR record, reset rhythm, increase in rhythm frequency, and tonic depolarization.

In spinal dogfish, cutaneous stimulation evokes reflex responses that are strongly dependent on the phase of the stimulus in the swimming cycle (Grillner et al., 1977). Here the responses are accompanied by augmented motor output and an instantaneous increase in swimming speed, as we have found in the immobilized Xenopus embryo.

Similar circuitry may also function in the mammalian spinal cord. Although direct evidence is still lacking, strong circumstantial evidence supports the idea that excitatory interneurons are included in the pathways from cutaneous afferents to limb motoneurons in the cat. The facilitation of cutaneous excitation in cat flexor digitorum longus motoneurons during the "active phase" of fictive locomotion (Schmidt et al., 1988) can best be explained by the inclusion of rhythmically active "last-order" excitatory interneurons in the pathways between afferents and motoneurons. Furthermore, Moschovakis et al. (1991) have demonstrated that the polysynaptic cutaneous excitation of the cat flexor digitorum longus motoneurons is greatly facilitated during the active phase of locomotion compared with more direct cutaneous inputs from the same source. This finding implies that as in the Xenopus embryo (present results) cutaneous pathways involve two parallel routes for excitation to reach the motor system, the longer of which includes premotor excitatory interneurons. Schefchyk et al. (1990) have also reported similar findings in the cat hindlimb, where premotor interneurons that are rhythmically active during locomotion receive polysynaptic cutaneous excitation. Interestingly, the same study describes dorsally located interneurons that receive more direct afferent input, but that are not active during locomotion. The input to these interneurons is, however, reduced during locomotion, suggesting that like Xenopus DLC interneurons they are inhibited during locomotion. Thus, the organization of adult vertebrate spinal circuitry that integrates cutaneous information during locomotion seems to be very similar to that which we have been able to demonstrate in the simple nervous system of a lower vertebrate embryo.

The organization of the spinal circuitry for phase-dependent reflex modulation in Xenopus embryos is remarkably similar to that which has been described recently in flying locusts. Phasedependent corrections to locust flight following deviations from the flight path are achieved by descending interneurons that relay excitation to flight motoneurons both directly and also in parallel through premotor interneurons (Reichert and Rowell, 1985; Reichert et al., 1985). Since the premotor interneurons 
A

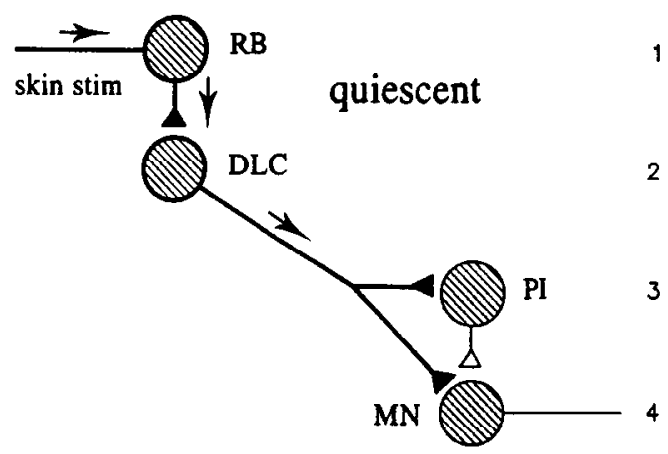

B

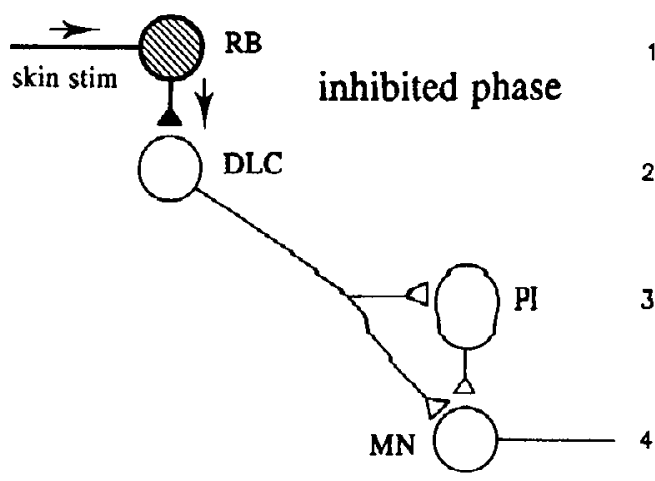

C

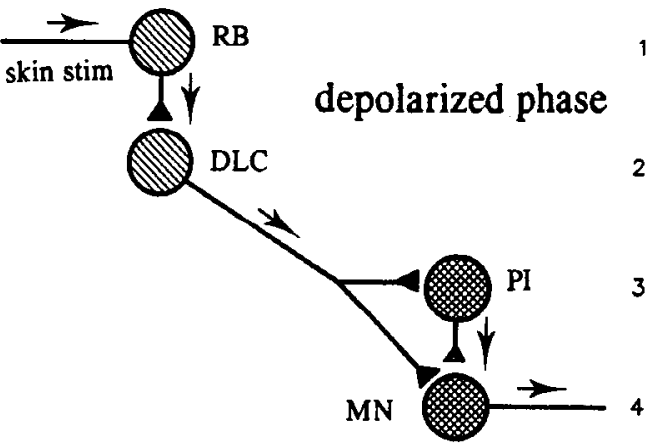

Figure 11. Circuit for excitatory crossed reflex pathway and its operation during fictive swimming. In each diagram, stimulus is given to left skin just above threshold for DLC impulse; circles represent neuron classes with axons and excitatory synapses (triangles, solid when active); heavy lines show when neurons or axons fire an impulse (arrows); shading shows the state of the neuron (hatching, at rest; open, inhibited; cross-hatching, excited). $A$, In quiescent embryo when neuron sits at resting potential. Stimulus excites sensory Rohon-Beard $(R B)$ neurons, which activate sensory interneurons $(D L C)$. These produce subthreshold EPSP simultaneously in premotor interneurons $(P I)$ and motoneurons $(M N)$ on the opposite side. Synapse from $P I$ to $M N$ is inactive. $B$, Same stimulus during swimming evokes no response if delivered in inhibited phase of $P I$ and $M N$ activity on opposite side, as crossed pathway blocked by simultaneous inhibitory gating signal onto DLC. $C$, In depolarized phase of opposite side $P I$ and $M N$ swim cycle (cross-hatching), inhibition of DLC is weak so crossed pathway is open so stimulus evokes extra action potential in opposite $P I$ and $M N$, which are both close to threshold. Connection from $P I$ to $M N$ (Dale and Roberts, 1985) is now also active so crossed excitation to $M N$ will be enhanced by indirect pathway. are themselves rhythmically active during flight, the descending information is phasically gated by alternating postsynaptic excitation and inhibition.

\section{References}

Baev KV, Kostyuk PG (1982) Polarization of primary afferent terminals of lumbosacral cord elicited by the activity of spinal locomotor rhythm generator. J Neurosci 2:1402-1409.

Bässler U (1976) Reversal of a reflex to a single motorneurone in the stick insect Carausius morosus. Biol Cybern 24:47-49.

Clarke JDW, Roberts A (1984) Interneurones in the Xenopus embryo spinal cord: sensory excitation and activity during swimming. J Physiol (Lond) 354:345-362.

Clarke JDW, Hayes BP, Hunt SP, Roberts A (1984) Sensory physiology, anatomy and immunohistochemistry of Rohon-Beard neurones in the spinal cord of Xenopus laevis. J Physiol (Lond) 348:511525 .

Currie SN, Stein PSG (1989) Interruptions of fictive scratch motor rhythms by activation of cutaneous flexion reflex afferents in the turtle. J Neurosci 9:488-499.

Dale N (1985) Reciprocal inhibitory interneurones in the spinal cord of Xenopus laevis. J Physiol (Lond) 363:527-543.

Dale N, Roberts A (1985) Dual component amino acid mediated synaptic potentials: excitatory drive for swimming in Xenopus embryos. J Physiol (Lond) 363:35-59.

Dale N, Ottersen OP, Roberts A, Storm-Mathisen J (1986) Inhibitory neurones of a motor pattern generator in Xenopus revealed by antibodies to glycine. Nature $324: 255-257$.

Davidoff RA (1972) The effects of bicuculline on the isolated spinal cord of the frog. Exp Neurol 35:179-19'3.

DiCapprio RA, Clarac F (1981) Reversal of a walking leg reflex elicited by a muscle receptor. J Exp Biol 90:197-203.

Dubuc R, Cabelguen J-M, Rossignol S (1988) Rhythmic fluctuations of dorsal root potentials and antidromic discharges of primary afferents during fictive locomotion in the cat. J Neurophysiol 60:2014 2036.

Duysens J (1977) Reflex control of locomotion as revealed by stimulation of cutaneous afferents in spontaneously walking premammillary cats. J Neurophysiol 40:737-751.

Duysens J, Trippel M, Horstmann GA, Dietz V (1990) Gating and reversal of reflexes in ankle muscles during human walking. Exp Brain Res 82:351-358.

Eccles JC, Schmidt R, Willis WD (1963) Depolarization of central terminals of group $1 \mathrm{~b}$ afferents of muscle. J Physiol (Lond) 168:500530.

Feldman AG, Orlovsky GN (1975) Activity of interneurons mediating reciprocal la inhibition during locomotion. Brain Res 84:181-194.

Forssberg H, Grillner S, Rossignol S (1975) Phase dependent reflex reversal during walking in chronic spinal cats. Brain Res 85:103-107.

Forssberg H, Grillner S, Rossignol S (1977) Phasic gain control of reflexes from the dorsum of the paw during spinal locomotion. Brain Res 132:121-139.

Grillner S, Rossignol S, Wallén P (1977) The adaptation of a reflex response to the ongoing phase of locomotion in fish. Exp Brain Res 30:1-11.

Hughes AFW (1957) The development of the primary sensory system in Xenopus laevis. J Anat 91:323-338.

Jordan LM (1983) Factors determining motoneuron rhythmicity during fictive locomotion. Soc Exp Biol Symp 37:423-444.

Kahn JA, Roberts A (1982) The central nervous origin of the swimming motor pattern in embryos of Xenopus laevis. J Exp Biol 99:185196.

Lennard PR, Hermanson JW (1987) Central reflex modulation during locomotion. Trends Neurosci 8:483-487.

Moschovakis AK, Sholomenko GN, Burke RE (1991) Differential control of short latency cutaneous excitation in cat digitorum longus motorneurones during fictive locomotion. Exp Brain Res 83:489-501.

Nieuwkoop PD; Faber J (1956) Normal tables of Xenopus laevis (Daudin). Amsterdam: North-Holland.

Pratt CA, Jordan LM (1987) 1a inhibitory interneurones and Renshaw cells as contributors to the spinal mechanism of fictive locomotion. J Neurophysiol 57:56-71.

Reichert H, Rowell CHF (1985) Integration of nonphaselocked ex- 
teroceptive information in the control of rhythmic flight in the locust. J Neurophysiol 53:1201-1217.

Reichert H, Rowell CHF, Gris C (1985) Course correction circuitry translates feature detection into hehavioural action in flying locusts. Nature 315:142-144.

Roberts A (1978) Pineal eye and behaviour in Xenopus tadpoles. Nature 273:774-775.

Roberts A, Alford ST (1986) Descending projections and excitation during fictive swimming in Xenopus embryos: neuroanatomy and lesion experiments. J Comp Neurol 250:253-261.

Roberts A Clarke JDW (1982) The neuroanatomy of an amphibian embryo spinal cord. Philos Trans R Soc Lond [Biol] 296:195-212.

Roberts A, Sillar KT (1990) Characterization and function of spinal excitatory interneurones with commissural projections in Xenopus laevis embryos. Eur J Neurosci 2:1051-1062.

Roberts A, Soffe SR, Dale N (1986) Spinal interneurones and swimming in frog embryos. In: Neurobiology of vertebrate locomotion (Grillner S, et al., eds), pp 279-306. London: Macmillan.

Roberts A, Dale N, Ottersen OP, Storm-Mathisen J (1988) Development and characterization of commissural interneurons in the spinal cord of Xenopus laevis embryos revealed by antibodies to glycine. Development 103:447-461.

Schefchyk S, McCrea D, Kriellaars D, Fortier P, Jordan L (1990) Activity of interneurones within the $\mathrm{L} 4$ spinal segment of the cat during brainstem-evoked fictive locomotion. Exp Brain Res 80:290-295

Schmidt BJ, Meyers DER, Fleshman JW, Tokuriki M, Burke RE (1988) Phasic modulation of short latency cutaneous excitation in flexor digitorum longus motoneurons during fictive locomotion. Exp Brain Res 71:568-578.

Schomburg ED, Behrends HB (1978) Phasic control of the transmission in the excitatory and inhibitory reflex pathways from cutaneous afferents to alpha-motoneurones during fictive locomotion in cats. Neurosci Lett 8:277-282.
Schomburg ED, Steffens H (1988) Rhythmic motor activity and phase dependent reflex transmission in the spinal tortoise (Testudo graeca, T. hermani). Neurosci Lett 8:277-282.

Sillar KT (1989) Synaptic modulation of cutaneous pathways in the vertebrate spinal cord. Sem Neurosci 1:45-54.

Sillar KT, Roberts A (1988a) A neuronal mechanism for sensory gating during locomotion in a vertebratc. Naturc 331:262-265.

Sillar KT, Roberts A (1988b) Unmyelinated cutaneous afferent neurones activate two types of excitatory amino acid receptor in the spinal cord of Xenopus laevis embryos. J Neurosci 8:1350-1360.

Sillar KT, Skorupski P (1986) Central input to primary afferent neurones in crayfish, Pacifastacus leniusculus, is correlated with rhythmic motor output of thoracic ganglia. J Neurophysiol 55:678-688.

Skorupski P, Sillar KT (1986) Phase-dependent reversal of reflexes mediated by the thoracocoxal muscle receptor organ in the crayfish, Pacifastacus leniusculus. J Neurophysiol 55:689-695.

Soffe SR (1987) Ionic and pharmacological properties of reciprocal inhibition in Xenopus cmbryo motoncurones. J Physiol (Lond) 382: 463-473.

Soffe SR, Clarke JDW, Roberts A (1984) Activity of commmissural interneurones in the spinal cord of Xenopus embryos. J Neurophysiol 51:1257-1267.

Stehouwer DJ, Farel PB (1981) Sensory interactions with a central motor program in anuran larvae. Brain Res 218:131-140.

Stein RB, Capaday C (1988) The modulation of human reflexes during functional motor tasks. Trends Neurosci 7:328-332.

Stewart WW (1978) Functional connections between cells as revealed with a highly fluorescent naphthalimide tracer. Cell 14:741-751.

Wallén $\Gamma$, Lansner A (1984) Do the motoneurones constitute a part of the spinal network generating the swimming rhythm in the lamprey. J Exp Biol 113:493-497.

Weevers $R$ de $G$ (1972) A simple and compact piezo-electric vibrator for microelectrode insertion. J Physiol (Lond) 303:22P. 